\title{
Búsqueda del Reservorio Marsupial (Didelphis marsupialis) de la Enfermedad de Chagas en el Estado de Hidalgo, México

\author{
Search of the Marsupial Reservoir (Didelphis marsupialis) of Chagas Disease in the State of \\ Hidalgo, Mexico
}

\author{
Imbert-Palafox $J^{1}{ }^{1}$, Tovar-Tomas $M R^{2}$, Peña-Rojas $A^{3}$, Becerril-Flores $M A^{4}$, Molina- \\ Trinidad $\mathrm{EM}^{5}$, Piña-Ruiz $\mathrm{HA}^{6}$ y Ruvalcaba Ledezma $\mathrm{JC}^{7}$
}

\begin{abstract}
:
Zoonoses are infections and diseases that are naturally transmitted from vertebrate animals to man and vice versa, where at least one domestic or wild animal species is involved, as is the case of the parasitic zoonosis caused by Trypanosoma cruzi, which has a transmitting vector as Triatominos through the participation of domestic or wild animal reservoirs. To determine, through a survey instrument, if the Tlacuache is an animal reservoir that probably maintains the biological cycle and the Chagas disease transmission and if it is in cohabitation with inhabitants of the State of Hidalgo. A cross-sectional descriptive observational study was carried out by applying a survey to 176 people. The instrument was elaborated by 10 questions about the reservoir commonly named "Tlacuache". It was also requested that photographic evidence of these mammals be obtained whenever possible and it was requested that, if possible, the hearts be obtained to perform the search for macroscopic pathology and the presence of the parasite in the hearts of opossums for the search of nests of amastigotes in the myocardium collected. Of the 176 respondents, $98.90 \%$ know the opossums. $76 \%$ of people use it for food purposes, $14.70 \%$ do not know, $7.84 \%$ use it for healing purposes and $0.98 \%$ use it as a pet. The results show a synanthropic relationship. Findings in hearts denote the effect of Tripanozoma spp. The results allow establishing that the opossum is a probable reservoir of the parasite Trypanosoma cruzi in the State of Hidalgo, which exists a synanthropic relationship with the communities. Situation that is not as different as the rest of the country's states.
\end{abstract}

Keywords:

Marsupial reservoir, Triatominos, Trypanosoma cruzi, Synanthropic

Resumen:

\footnotetext{
'Autor de correspondencia. Universidad Autónoma del Estado de Hidalgo. Instituto de Ciencias de la Salud. Pachuca, Hidalgo, México. Email: imbertox@hotmail.com

2 Universidad Autónoma del Estado de Hidalgo. Instituto de Ciencias de la Salud. Pachuca, Hidalgo, México. Email: mari_blue19@hotmail.com

Universidad Autónoma del Estado de Hidalgo. Instituto de Ciencias Agropecuarias. Pachuca, Hidalgo, México. Email: angelpr965@gmail.com

4 Universidad Autónoma del Estado de Hidalgo. Instituto de Ciencias de la Salud. Pachuca, Hidalgo, México. Email: mbecerril_65@yahoo.com

Universidad Autónoma del Estado de Hidalgo. Instituto de Ciencias de la Salud. Pachuca, Hidalgo, México. Email: mariaeva_molina@yahoo.com.mx

6 Universidad Autónoma de Yucatán. Centro de Investigación Regional Dr. Hideyo Noguchi. Yucatán, México. Email: tlacuayuca@gmail.com

${ }^{7}$ Universidad Autónoma del Estado de Hidalgo. Instituto de Ciencias de la Salud. Pachuca, Hidalgo, México. Email: 
Las zoonosis son enfermedades e infecciones que se transmiten naturalmente de los animales vertebrados al hombre y viceversa, donde se involucra por lo menos una especie animal doméstica o silvestre, como es el caso de la zoonosis parasitaria causada por Trypanosoma cruzi, que tiene como vector transmisor a Triatominos mediante la participación de reservorios animales domésticos $o$ silvestres. Para determinar si el Tlacuache es un reservorio animal que probablemente mantiene el ciclo biológico y la transmisión de la enfermedad de Chagas y si se encuentra en convivencia con habitantes del Estado de Hidalgo se utilizó una encuesta. Se realizó un estudio observacional descriptivo de carácter transversal mediante la aplicación de una encuesta a 176 personas. El instrumento se elaboró conformado por 10 preguntas sobre el reservorio nombrado comúnmente como "Tlacuache". Se solicitó además que cuando fuera posible se obtuvieran evidencias fotográficas de estos mamíferos y se solicitó que en caso de que fuera posible, se obtuvieran los corazones para realizar la búsqueda de patología macroscópica y la presencia del parasito en los corazones de tlacuaches para la búsqueda de nidos de amastigotes en los miocardios colectados. De los 176 encuestados, el $98.90 \%$ conoce los tlacuaches. El $76 \%$ de la gente lo usa para fines alimenticios, el $14.70 \%$ no sabe, el $7.84 \%$ lo ocupa para fines curativos y para el $0.98 \%$ se emplea como mascota. Los resultados muestran una relación sinantrópica. Los hallazgos en los corazones denotan efecto de Tripanozoma sp. Los resultados permiten establecer que el tlacuache es probable reservorio del parasito Trypanosoma cruzi en el Estado de Hidalgo y que existe una relación sinantrópica con las comunidades. Situación que no es tan diferente como al resto de los estados del país.

\section{Palabras Clave:}

Reservorio marsupial, Triatominos, Trypanosoma cruzi, sinantrópico

\section{Introducción}

La Organización Mundial de la Salud definió desde 1959 el término de "Zoonosis" como "las enfermedades e infecciones que se transmiten naturalmente de los animales vertebrados al hombre y viceversa". En estos ciclos biológicos y de transmisión de las enfermedades se involucra por lo menos a una especie animal doméstica o silvestre [1]. Considerando este aspecto, la tripanosomiasis americana (o enfermedad de Chagas) es una zoonosis parasitaria causada por Trypanosoma cruzi, que tiene como vector transmisor a los insectos conocidos como chinches y que mantiene en forma perpetua el ciclo mediante la participación de reservorios animales domésticos o silvestres. Considerado desde otro concepto, esta relación con los animales no domesticados de convivencia con el entorno humano, se denomina Sinantropia, (del griego syn -, "junto a" + anthro, "humano"), y se aplica a especies de plantas y animales silvestres que viven cerca y se benefician de los habitats artificiales creados por los humanos a su alrededor [2, 3, 4]. Como sinantropismo se entiende entonces la invasión de plagas a la comunidad ecológica dominada por los humanos, en la cual coexisten por largos periodos de tiempo. El grupo de mamíferos silvestres que mejor ejemplifica este concepto de sinantropía es el de los marsupiales del genero Didelphis, mejor conocidos como zarigüeyas, gambás, zorros 0 tlacuaches. Esta relación desde luego genera condiciones de riesgo para la transmisión de agentes zoonoticos, ya que son una fuente natural de patógenos para los habitantes [5, 6, 7].

En este aspecto, los "reservorios" o eslabones sinantrópicos de la enfermedad de chagas en el estado de Hidalgo no se han identificado. A pesar de que se tienen registros de los vectores transmisores dentro del ciclo de la enfermedad (Figura 1); poco a poco se han identificado a los que tienen el papel de ser la fuente 0 reserva, y son denominados animales sinantrópicos y desde luego que también es importante y necesario conocerlos (conocidos también como "host mammal species" o especies de mamíferos hospederos). Sobre todo es importante ahora que se empieza a cuestionar la ruta estercórea y el papel del vector como la principal causa de transmisión de la enfermedad al humano, pues se está considerando que hay un eslabón perdido en la dinámica y el control de esta enfermedad [8]. La baja probabilidad de transmisión por vía vectorial de " $5.8 \times 10$ " 4", deducida de datos entomológicos básicos, refleja muy bien el complejo y no tan frecuente mecanismo de transmisión vía las heces del insecto. Más que nada, considerando a la gran mayoría de agentes causales de enfermedades transmitidas por vector como dengue, malaria, ulceras y otras de flavivirus cuya ruta es a través de la saliva o "salivaría" y cuya probabilidad de transmisión es más alta, por ejemplo 0.5 a 1 por picadura para malaria [8].

Los estudios realizados sobre este aspecto señalan como reservorios naturales de Trypanosoma cruzi a varios animales mamíferos dentro de varios ordenes como roedores, marsupiales, armadillos, murciélagos y primates silvestres además de ciertos animales domésticos como perros, gatos e incluso ratas y cobayos $[9,10]$.

Figura 1. Ciclo biológico de la Enfermedad de Chagas.

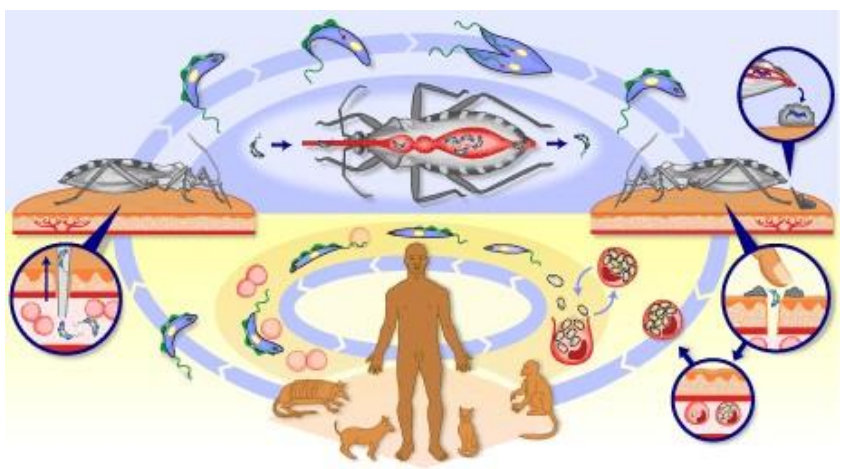

A pesar de que en la cultura de la población del estado de Hidalgo se conoce en general la existencia del tlacuache, no hay suficientes registros científicos 0 
formales que así lo demuestran (aunque artísticos si los hay: en el mural que se encuentra en el teatro "Gota de Plata, en Pachuca, Hidalgo; se muestra a un tlacuache en el centro de la obra, Figura 2).

Figura 2. Mural en el teatro Gota de Plata, en Pachuca Hidalgo, México.

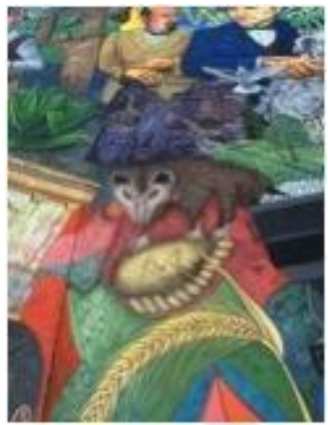

Hay algunos estudios con escasos resultados que rescatan este aspecto como: "no existe un inventario actualizado y completo de los mamíferos que habitan el PNEC; la riqueza mastozoológica del estado de Hidalgo es poco conocida [9], lo que lo ha llevado a ocupar el $17^{\circ}$ lugar en el país". Se puede mencionar que en el estado de Hidalgo se han registrado un total de 155 especies comprendidas en 81 géneros, 21 familias y ocho órdenes. Los órdenes mejor representados son el Chiroptera (murciélagos) con 67 especies (43.23\%) y el Rodentia (roedores) con 46 especies (29.67\%). Con relación a su distribución, las especies de mamíferos de Hidalgo se localizan en 33 de 84 municipios [10]. (El Orden Chiroptera es el mejor representado con 28 especies (más 2 a nivel de género, por falta de cráneo), le siguen el Orden Rodentia con 12 especies (más 3 a nivel de género, por falta de cráneo) y finalmente el Orden Carnivora con 11 especies. Los órdenes menor representados son el Didelphimorphia, con cuatro especies; Soricomorpha y Artiodactyla con dos especies cada una y Cingulata, Pilosa y Lagomorpha con un representante a nivel de especie cada uno [9]. Sin embargo, de acuerdo a la Comisión Nacional de la Biodiversidad (CONABIO), la distribución potencial espacial de este reservorio animal es muy amplia no solo en el estado de Hidalgo sino en todo el país [11], (ver el mapa).

Link:

http://www.conabio.gob.mx/informacion/gis/layouts/did_vi rggw.png).

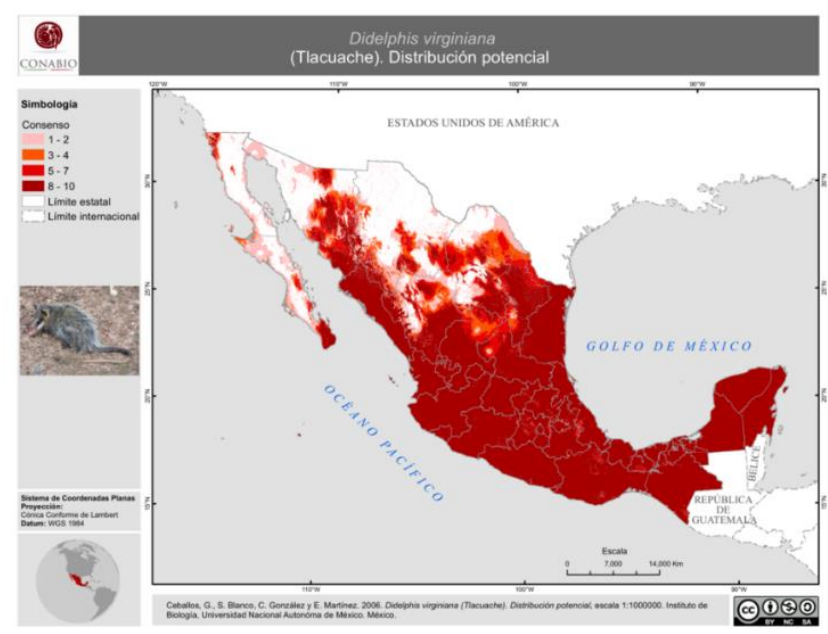

El no considerar a los eslabones domésticos y silvestres en la "red o network" de la enfermedad de Chagas como el perro, gato, armadillo y monos como hospederos, en general simplifica la situación e impide otras perspectivas y acciones, sobre todo en la salud Publica. Actualmente también se deben considerar a otras especies dentro del concepto de reservorios como la rata, el ratón, tlacuaches, los zorros e inclusive los murciélagos y en general animales de sangre caliente [12].

El costo-efectividad, las estrategias de control de vectores y la prevención de la transmisión de la enfermedad de Chagas requiere considerar el potencial continuo del movimiento de los parásitos en el panorama entero. En este estudio que se cita, realizado en el estado de Morelos (6), (entidad con reportes de alta infestación de vectores, infecciones humanas y enfermedad de Chagas rodeado por áreas agrícolas y naturales muy similar a nuestro estado), se proveen claras evidencias de que esas estrategias requieren incluir las especies reservorias que se encuentran en los "hábitats" silvestres, ecológicos y domésticos [5,7]. Se debe considerar nuevos indicadores como el IRC (índice de reservorio de Chagas), el cual mide la importancia relativa de los hospederos de los triatomas como reservorios competentes de $T$. cruzi asociados con la infección humana [5]. Debido a lo anterior, es que es importante conocer y ubicar las fuentes o "reservas" del parasito en el ciclo biológico completo y complejo en el que la población del estado de Hidalgo está expuesta, confirmar si interactúan e incluso si se modifican directamente o indirectamente a la comunidad de reservorios. Por lo tanto, el objetivo del trabajo fue determinar a través de un instrumento de encuesta, si el Tlacuache, como un reservorio animal que probablemente mantiene el ciclo biológico y la transmisión de la enfermedad de chagas se encuentra en convivencia con habitantes del Estado de Hidalgo.

\section{Metodología}

Se realizó un estudio observacional, descriptivo, de carácter transversal mediante la aplicación de una encuesta. El instrumento se elaboró conformado por 10 
preguntas sobre el reservorio nombrado comúnmente como "Tlacuache".

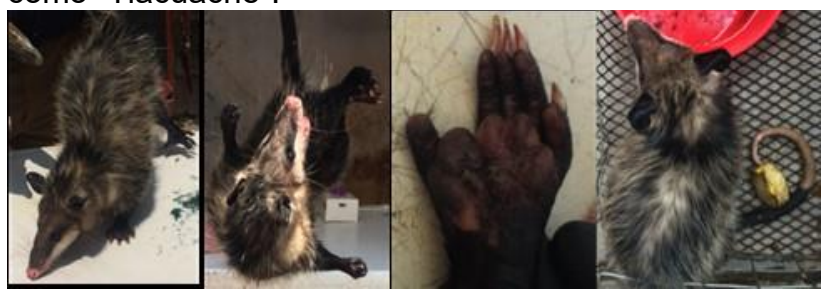

Se imprimieron 200 ejemplares y se entregaron cinco o diez por alumno. Fue aplicado por alumnos del tercer semestre de la escuela de medicina, durante un periodo de dos meses de marzo a junio del año 2014; la mayoría de los estudiantes son originarios de la entidad, y la encuesta se realizó a 176 personas de su entorno familiar y vecinal.

Se solicitó además que cuando fuera posible se obtuvieran evidencias fotográficas de estos mamíferos y se solicitó que en caso de que fuera posible, se colectaran corazones para realizar la búsqueda de patología macroscópica y encontrar al parasito en nidos de amastigotes, en dichos órganos, para lo cual se entregaron 20 recipientes de plástico con $200 \mathrm{ml}$ de formol-PBS $0.01 \mathrm{M}, \mathrm{pH} 7.4$, indicando que se entregaran a las personas que manifestaron que los cazan con fines alimenticios. Una vez trasladados al laboratorio se realizaron disección macroscópica y posteriormente se procesaron histológicamente con la técnica de Hematoxilina y Eosina, para buscar nidos de amastigotes en los miocardios.

De manera informal, los estudiantes entrevistaron a personas que dijeron conocer a los tlacuaches para conocer relatos o por hechos curiosos o anecdóticos y aqui se presenta uno de ellos.

\section{Resultados}

De 176 personas encuestadas el $61.78 \%$ son hombres y el $38.22 \%$ son mujeres. El rango de edad de los conocedores del tlacuache siguió una distribución normal, con un máximo en los 40 a 50 años. En los grupos etarios que fueron encuestados, el mayoritario correspondió al de los 41 a 50 años y los minoritarios al de la edad de 71 a 80 y 81 a 90 .

El instrumento indica que el $98.90 \%$ conoce los tlacuaches. El $76 \%$ de la gente lo usa para fines alimenticios, el $14.70 \%$ no sabe, el $7.84 \%$ lo ocupa para fines curativos y para el $0.98 \%$ se emplea como mascota. También se encontró que el $60.78 \%$ de la gente dice que los tlacuaches salen en la noche; el $21.57 \%$ en la tarde; el $11.77 \%$ en la madrugada y el $5.88 \%$ en la mañana. El instrumento una relación sinantrópica pues el $93.77 \%$ de las personas los ha visto cerca de su domicilio y el $6.33 \%$ no los ha visto cerca de su domicilio.

La distribución del reservorio fue en 12 municipios de Hidalgo (Apan, Ajacuba, Atotonilco el Grande, Huejutla de Reyes, Yahualica, Zacualtipan de Ángeles, Tulancingo de Bravo, Santiago de Anaya, Metztitlán, Zimapán, Zapotlán de Juárez, Mineral de la Reforma) y cuatro municipios de estados vecinos como Veracruz, San Luis Potosí y Querétaro (Tamazunchale, Xilitla, Tantoyuca y San Juan del Río). Las coordenadas geográficas N/O fueron $19^{\circ}$ a $21^{\circ}$ y $98^{\circ}$ a $99^{\circ}$ y la altitud la cual vario desde $15 \mathrm{msnm}$ hasta 2,505 msnm. Los datos muestran la presencia del reservorio en doce comunidades del Estado de Hidalgo que comprenden un tamaño de población de 686,478 habitantes o el $24 \%$ de la población total del estado $(2,858,359$ habitantes).

Figura 3. Fotografías de un joven tlacuache macho de Didelphis marsupialis, atrapado en Singuilucan, Hidalgo abajo fotografías de una hembra de Didelphis virginiana capturada en áreas suburbanas de la ciudad de Mérida, Yucatán.

Imbert PJL ${ }^{\circledR}$ CopyRight

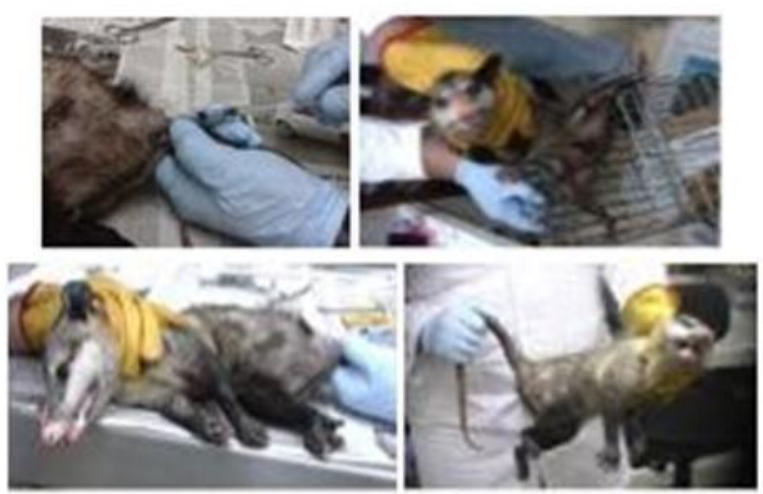

Figura 4. Tlacuaches encontrados en la cabecera municipal de Huejutla, Hidalgo (Hospital General de Zona, IMSS) y en

la colonia Cuauhtémoc Municipio de Actopan, Hidalgo. Tamaño aproximado de $80 \mathrm{~cm}$, tonalidad obscura grisácea del pelo y la cabeza y alrededor del hocico así como blanquecina del abdomen, aparentemente del género Didelphis marsupialis. El tercer Tlacuache, se encontró en la colonia de

Mangas, carretera los Hoyos en Tezontepec de Aldama,

Municipio del Estado de Hidalgo, zona conurbana a 4

kilómetros de la capital; el cuarto marsupial fue un macho (media $83.5 \mathrm{~cm}$ de largo, largo de cola $36 \mathrm{~cm}$, largo de cabeza

$13,5 \mathrm{~cm}$ y ancho de cabeza $4.5 \mathrm{~cm}$ ), encontrado por la PROFEPA del estado de México, y lo llevaron al parque de convivencias del cerro de cubitos en Pachuca, Hidalgo. Note que no es difícil encontrar semejanzas.

Imbert PJL ${ }^{\circledR}$ CopyRight 

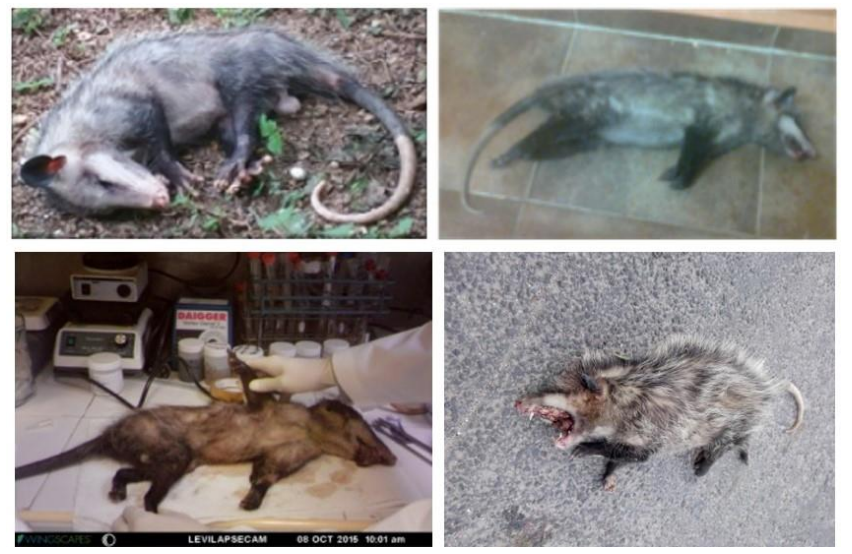

Figura 5. Anatomía de corazones de Tlacuaches del Estado de

Hidalgo. Arriba se muestra pared ventricular deforme,

adelgazamiento de paredes interiores, fibras nerviosas aisladas expuestas y aspecto translucido por luz que atraviesa el ventrículo muy delgado. En las de abajo, note las arterias y

venas externas que irrigan. La última muestra áreas

ventrículo-auriculares más densas, llenas de tejido sin

adelgazar y sin fibras nerviosas desnudas, aparentemente normales.

Fotografías Imbert PJL ${ }^{\circledR}$ CopyRight
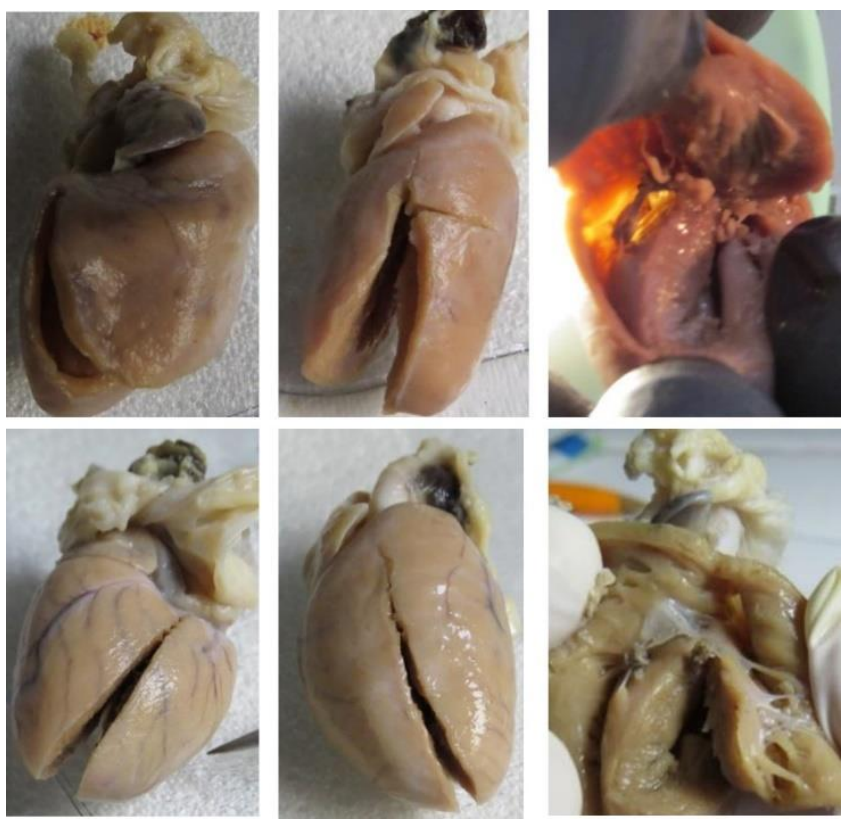

Figura 6. Cortes teñidos con Hematoxilina y Eosina de los corazones de Tlacuaches provenientes del Estado de Hidalgo, llevados al laboratorio en febrero del 2015. Se muestra el

tejido con un nido largo de amastigotes entre las fibras desorganizadas del miocardio y un nido menos característico;

abajo los nidos son largos y rodeados probablemente de colágeno y el tejido sin inflamación ni células inmunes. Todas las imágenes son a $40 \mathrm{X}$.

Fotografías Imbert PJL ${ }^{\circledR}$ CopyRight


\section{La narrativa del abuelo}

Se dice acerca del tlacuache (Entrevista con abuelo de alumna de medicina, de la UAEH).

En la comunidad de la colonia Cuauhtémoc Municipio de Actopan, Hidalgo ha disminuido la especie por el aumento de la caza de estos animales.

Octubre, noviembre, diciembre y enero: son los meses más aptos para la captura de un tlacuache.

Para poder cocinarlo se le queman los pelos y posteriormente se lava bien, después se realiza una incisión longitudinal a lo largo del esternón, se proceden a retirar intestinos y se enjuaga muy bien con agua para proceder a prepáralo para barbacoa que sería "tlacuache enchilado"

El tiempo que tarda en coserse el tlacuache, en horno para barbacoa es de 3 horas a más.

Dicen que si te llega a morder uno no te suelta $Y$ que la manera más fácil de agarrarlos es de la cola.

Duermen durante el día y en la noche caminan son como (caminantes) nómadas porque una noche están en un lado y otra noche en otro no tiene lugar fijo para establecerse, aunque tengan hijitos los andan cargando.

Por ejemplo las hembras tienen un morralito en la parte abdominal para poder introducir a sus bebes y no dejarlos, los andan cargando a todos los lugares a donde vayan ya sea de día o de noche.

Los lugares en donde pueden dormirse: son en lugares huecos, por ejemplo, en las raíces de un árbol o cualquier otro hueco.

Se alimentan de: maíz, aguamiel, huevo, lombrices, pollos

\section{Discusión}

En este estudio fue sorprendente conocer por primera vez una distribución parcial y estimar que hay una población real del tlacuache, probable reservorio del parasito Trypanosoma cruzi en el Estado de Hidalgo; y sobre todo confirmar que si hay una relación sinantrópica con las comunidades. Situación que no es tan diferente como al resto de los estados del país [5, 6, 7]. Las evidencias 
sobre la presencia de estos reservorios indican que la especie predominante en el estado es probablemente Didelphis marsupiales (Figura 3, arriba) pues el pelaje es diferente a los de Mérida, Yucatán Didelphis virginiana (Figura 3, abajo). Actualmente esta diferencia solo puede establecerse con biología molecular.

Sorprende aún más el conocer a través de la entrevista con la persona mayor, que la manera de prepararlo y el método de cocción para cocinarlo y comerlo como "barbacoa" no asegura que se elimine a los trypanosomas. Como se mencionó al principio, la transmisión por vía oral no solo se ha confirmado sino que está tomando otra perspectiva a la luz de nuevas investigaciones, que cuestionan que la mayor vía de contagio sea la del vector [8, 13], y probablemente la transmisión vía oral en mamíferos domésticos contribuye fuertemente a la dispersión de la enfermedad. En particular, provee de una alternativa a la picadura del vector como una ruta de infección para los animales domésticos.

La transmisión vía oral es posible a través de alimentos contaminados por las heces del vector, aunque se conocen muy poco las características epidemiológicas y clínicas de la enfermedad de Chagas adquirida en forma oral [14]. Al considerarla como una enfermedad que puede originar brotes epidémicos que son transmitidas por los alimentos [15], la ruta oral, es responsable de múltiples brotes en regiones o aéreas geográficas en las que se ha interrumpido la vía vectorial. Surge además otro aspecto que es importante conocer sobre los componentes fisiológicos, bioquímicos e inmunológicos de la posible interacción del parasito con la barrera placentaria o el tracto gastrointestinal [16].

Si consideramos la gran proporción de personas que realizan esta tradición de cazar, preparar, cocinar y comer a este y otros tipos de reservorios [17], (Link para ver la película y cartel donde se invita a un evento gastronómico anual que es parte de la cultura de muchas comunidades (https://www.youtube.com/watch?v=dXXF40zo72k), y reconsideramos esta posibilidad de transmisión [18], resultaría alarmante que fuera a través de este medio como se mantiene la endemicidad y por ende la morbimortalidad de esta enfermedad.

Finalmente, las figuras de los corazones de los tlacuaches, órgano blanco de los trypanosomas, donde se muestra la anormalidad anatómo-patológica principal de la enfermedad de Chagas, esto es el "adelgazamiento del ventriculo por la replicación del Trypanosoma y la exposición de las fibras nerviosas cuando se ha destruido las fibras musculares cardiacas" que dan origen a los registros electro-cardio-gráficos como las arritmias cardiacas y otros signos clínicos que indican daño al miocardio es sorprendente. La confirmación de la infección mostrando la presencia de diversos nidos de amastigotes en el tejido cardiaco de estos reservorios también lo es.

\section{Conclusiones}

Es importante la información geográfica obtenida de la distribución de este reservorio ya que ubica mejor las poblaciones, no solo para considerar un riesgo epidemiológico sino también para realizar otras investigaciones futuras que incluyan la búsqueda del parasito. Es decir, lo aquí encontrado, demanda realizar un objetivo posterior el cual sería determinar la frecuencia de tlacuaches infectados y confirmar si el parasito es Trypanosoma cruzi.

Si bien es cierto que en el mecanismo de contagio para la enfermedad de Chagas se considera la vía transmisión del vector, habría que poner atención a la vía oral o a través de la ingesta de animales, que son considerados como alimentos y que de una u otra manera representan reservorios naturales. De igual manera hay que considerar la ingesta de frutas contaminadas con las heces de las chinches besuconas, sobre todo porque se ha descubierto existe preferencia de estas por el azúcar de las frutas y que amplia de manera significativa la posibilidad de abrir puertas nuevas en investigación. Por lo pronto, un aspecto fundamental que resulta a partir de la información presentada en este documento, es la emergencia de trabajar aun mas en la educación y promoción de la salud en comunidades donde estos reservorios animales forman parte cultural de los alimentos emergentes en su dieta.

Agradecimientos. A la Secretaria de Educación Publica SEP: Programa de Mejora del Profesorado) por el financiamiento al proyecto "Red Epidemiologica de Enfermedades Zoonoticas y Transmitidas por Vector (Etv's) de Importancia en la Salud Pública", Convocatoria 2013 para la colaboracion de Redes Tematicas.

Etica de publicaciones. No hemos requerido informacion privada o intima acerca de la vida de las personas, familiar o costumbres y habitos y lo que se declaro es del conocimiento general de las comunidades. Los encuestadores fueron entrenados para no herir o dañar los sentimientos o la dignidad.

Consentimiento para participar. El consentimiento informado por escrito no fue necesario dado que se solicito permiso previo para participar en la encuesta.

Consentimiento para publicar. No es aplicable.

Conflicto de intereses. Los autores declaran que no hay conflcito de interes para publocar este articulo.

Disponibilidad de informacion. Cada una de las encuestas está disponible en forma impresa. Una copia electronica está disponible bajo requerimiento.

Contribucion de los autores. Imbert, PJL (Diseñó el instrumento de encuesta, organizo y coordino la investigacion y escribio el manuscrito inicial). Tovar TMR (Capturo datos y realizo las disecciones). Piña, RA (proceso los corazones y realizo las tecnicas histologicas). Becerril FMA (estudio las preparciones microscopicas y reviso la version final del manuscrito). Molina TEM (Corrigio las pruebas de galera y edito el manuscrito final). Piña RHA (propuso el tema y la idea original). Ruvalcaba LJC (Corrigio las pruebas de galera y edito el manuscrito final).

"Todos los autores leyeron y aprobaron la version final del manuscrito" 


\section{Referencias}

[1] WORLD HEALTH ORGANIZATION. (2018). Chagas disease (American trypanosomiasis). Accessed June 14, 2018, http://www.who.int/news-room/fact-sheets/detail/chagas-disease(american-trypanosomiasis)

[2] Ruiz-Piña HA, Cruz-Reyes A. The Opossum Didelphis virginiana as a Synanthropic Reservoir of Trypanosoma Cruziin Dzidzilché, Yucatán, México. Mem Inst Oswaldo Cruz. 2002; 97(5): 613-620.

[3] Pacheco CJ, Lugo PJA, Tzuc CL, Ruiz PHA. Capítulo 8. Mamíferos Sinantrópicos y la Transmisión de Enfermedades Zoonoticas en el Area Rural de Yucatan. Capitulo 10. El "zorro" de Yucatán y su Relacion con la Poblacion Humana. En: Estudios Multidisciplinarios de las Enfermedades Zoonoticas y ETVs en Yucatan. 2013; Ed. Universidad Autónoma de Yucatan. Merida, Yucatan, México. 283pp. ISBN: 978607-8191-65-9.

[4] McFarlane R, Sleigh A, McMichael. Synanthropy of Wild Mammals as a Determinant of Emerging Infectious Diseases in the AsianAustralasian Region. EcoHealth. 2012; 9, 24-35. DOI: 10.1007/s10393012-0763-9

[5] Ramsey JM, Gutiérrez-Cabrera AE, Salgado-Ramírez L, Townsend Peterson A, Sánchez-Cordero V, Ibarra-Cerdeña CN. Ecological Connectivity of Trypanosoma CruziReservoirs and Triatoma pallidipennis Hosts in an Anthropogenic Landscape with Endemic Chagas Disease. PLoS ONE. 2012; 7(9): e46013. doi:10.1371/journal.pone.0046013.

[6] Hernández FM, Franco ER, Campillo LMG, García CV, Valdovinos MR, Maravilla P, Aguilar RA, Rivas N, Aguilar AC, García CIM and Villalobos G. Follow up of natural infection with Trypanosoma Cruziin two mammals species, Nasua narica and Procyon lotor (Carnivora: Procyonidae): evidence of infection control?. Parasites \& Vectors. 2014; 7:405

[7] Villagrán ME, Martínez-Ibarra JA, de Diego JA. Pathological alterations and prevalence of Trypanosoma Cruziin opossums from western Mexico. Boletín de Malariología y Salud Ambiental. 2011; 1 (1): $87-88$

[8] Nuovellet P, Dumonteil E, Gourbiére S. The Improbable Transmission of Trypanosoma Cruzito Human: The missing link in the Dynamics and Control of Chagas Disease. Plos. Neglected Tropical Diseases. 2013; 7(11)e2505.

[9] Hernández-Flores SD, Rojas-Martínez AE. Lista Actualizada y Estado de Conservación de los Mamíferos del Parque Nacional El Chico, Hidalgo, México. Acta Zoológica Mexicana (n.s.) 2010; 26(3): 563-583.

[10] Mejenes-López SMA, Lorenzo-Monterrubio C. Mastofauna de la Región Huasteca y Sierra de Hidalgo. Tesis Biología. Universidad Nacional Autónoma de México. 2008. repositorio.fciencias.unam.mx:8080/jspui/bitstream/11154/...PDF\%20 unificado.pdf

[11] Ceballos G, Blanco S, González C and Martínez E. 'Didelphis virginiana (Opossum). Distribución potencial'. Extraído del proyecto DS006 'Modelado de la distribución de las especies de mamíferos de México para un análisis GAP'. Con un tamaño de píxel: 0.01 grados decimales. Instituto de Biología, Universidad Nacional Autónoma de México (UNAM), (2006). Financiado por la Comisión Nacional para el Conocimiento y Uso de la Biodiversidad (CONABIO). México.

[12] Ibarra-Cerdeña CN, Sánchez-Cordero V, Townsend-Peterson A, Ramsey JM. Ecology of North American Triatominae. Acta Tropica. 2009; 110:178-186.

[13] Coffield Jr DJ, Spagnuolo AM, Shillor AM, Mema M, Pell E, Pruzinsky B, Zetye A. A Model for Chagas Disease with Oral and Congenital Transmission. PLoS ONE, 2013; 8(6):e67267. doi:10.1371/journal.pone.0067267

[14] de Noya BA, Díaz ZB, Colmenares C, Ruiz RG, Mauriello L, Zavala RJ, Suarez JA, Abate T, Naranjo L, Paiva M, Rivas L, Castro J, Márques J, Mendoza I, Acquatella H, Torres J. and Noya O. Large
Urban Outbreak of Orally Acquired Acute Chagas Disease at a School in Caracas, Venezuela. The Journal of Infectious Diseases. 2010; 201(9):1308-1315.

[15] Pereira KS, Schmidt FL, Guaraldo AMA, Franco RMB, Dias VL. and Passos LAC. Chagas' Disease as a Foodborne Illness. Journal of Food Protection. 2009; 72(2): 441-446.

[16] Sánchez LV and Ramírez JD. Congenital and oral transmission of American trypanosomiasis: an overview of physiopathogenic aspects. Parasitology / September 2012, pp 1-13. DOI: 10.1017/S0031182012001394.

[17] Cantillo-Barraza O, Garcés E, Gómez-Palacio A, Cortés LA, Pereira A, Marcet PL, Jansen AM and Triana-Chávez O. Ecoepidemiological study of an endemic Chagas disease region in northern Colombia reveals the importance of Triatoma maculata (Hemiptera: Reduviidae), dogs and Didelphis marsupialis in Trypanosoma Cruzimaintenance. Parasites \& Vectors. 2015; 8:482. DOI $10.1186 / \mathrm{s} 13071-015-1100-2$

[18] Melissa N, Garcia MN, Murphy SK, Gross A, Wagner J and Murray KO. Knowledge, attitudes, and practices of Texas hunters: a potentially high-risk population for exposure to the parasite that causes Chagas disease. Parasites \& Vectors. 2015; 8:197. 